\title{
Modeling ultrafast transitions within laser-irradiated solids
}

\author{
B. Ziaja ${ }^{* 1,2}$ and N. Medvedev ${ }^{1}$ \\ ${ }^{1}$ Center for Free-Electron Laser Science, \\ Deutsches Elektronen-Synchrotron, \\ Notkestrasse 85, D-22607 Hamburg, Germany \\ ${ }^{2}$ Institute of Nuclear Physics, Polish Academy of Sciences, \\ Radzikowskiego 152, 31-342 Kraków, Poland
}

(Dated: November 7, 2011)

\begin{abstract}
We give an overview of physical mechanisms contributing to radiation induced changes within solids irradiated with femtosecond X-ray FEL pulses and present some models to describe the evolution of the irradiated samples. Applicability of these models is then discussed with respect to the structure of an irradiated material and to the pulse parameters.
\end{abstract}

Keywords: free-electron lasers; ultrafast laser-induced transitions; solids

PACS numbers: 41.60.Cr, 52.50.Jm, 52.30.-q, 52.65.-y

* Corresponding author. E-mail: ziaja@mail.desy.de 


\section{INTRODUCTION}

In order to describe ultrafast transformations in solids induced by intense XFEL radiation (LCLS, SACLA, European XFEL [1-3]), such as changes of optical properties, magnetic properties or the formation of defects, we need to understand the fundamental processes on a microscopic level. This requires the development of dedicated theoretical approaches. These tools can be based on particle or kinetic approaches, the applicability of which will depend on the sample size, structure and its physical regime (quantum, classical). These tools usually apply techniques such as: Monte-Carlo (MC) [4], molecular dynamics (MD) $[5,6]$, hydrodynamic or kinetic equations [7-9] or some combination of these approaches.

$\mathrm{X}$-ray photons initiate various processes in solids. Photons can scatter elastically from atoms and ions, or can ionize them. Photoionization at these photon energies primarily releases electrons from inner shells ( $\sim 95 \%$ for light elements). The subsequent relaxation is predominantly follows, for light elements, affected by Auger decays. In such cases a single photoionization event ends up with two electrons released: a high energy photoelectron of the energy comparable to the X-ray photon, and an Auger electron, with energy of the order of a few hundreds eVs [10].

Next, the liberated electrons propagating in the solid interact with the constituent atoms. These interactions may be either inelastic or elastic. The average distance travelled by an electron between two consecutive inelastic or elastic collisions is described by a mean free path, $\lambda_{i n(e l)}$, which is proportional to the inverse of the inelastic or elastic cross section.

During a collision the impact electron loses a part of its energy, transferring it to electron(s) or to the lattice. There are several energy loss channels accessible for the primary electron: (i) direct production of electron-hole pairs during core or valence ionizations, (ii) collective excitations of the solid (plasmons, excitons and phonons), [11-13]. The accessibility of the loss channels depends on the energy of the primary electron, $E$, measured with respect to the bottom of the conduction band. If $E$ is larger than the threshold for the inner shell (core) or valence ionization, all routes for the energy loss are available for the primary electron, and the direct production of electron-hole pairs is the most likely route. The electron will be able to ionize any electron whose ionization energy is lower than the primary electron energy.

If the energy of the primary impact lies below the threshold for plasmon excitations, 
$E<E_{P}$, the plasmonic channel for energy loss closes. At even lower energies, the production of electron-hole pairs will be suppressed. This occurs at energies, $E \leq E_{G}$, where $E_{G}$ is the band gap width. For metals the direct production of electron-hole pairs will be possible at any impact energy, $E>E_{F}$, where $E_{F}$ is the Fermi energy.

In both semiconductors and insulators long-living excitations of bound electron-hole pairs, called excitons, are also possible. The thresholds for excitons lie around $E_{G}$. At energies below exciton thresholds only phonon excitations occur with the energy gains or losses of less than $0.1 \mathrm{eV}$. Phonons may also be excited in metals. Due to the small energy transfer required for a phonon exchange, the phononic channel for energy loss remains open even at very low impact energies both in metals and in the semiconductors.

Electrons of low energies, $E_{G}\left(E_{F}\right)<E<E_{P}$, moving in solids experience strong electronelectron scattering processes. In metals, inelastic lifetimes of these electrons, $\tau$, have been studied for many years in the framework of the free-electron-gas model $[11,12]$. However, recent experiments and calculations $[14,15]$ have shown that the band structure of metals is very important for the electron transport at low electron energies. The calculations have shown that the lifetimes of the electrons are strongly affected by the topology of the Fermi surface and the density of states in bands, even for free-electron-like metals for which the free-electron-gas model works with good accuracy, e.g. aluminium, see Ref. [12, 16, 17].

The details of the band structure will strongly effect the electron scattering, which was observed in semiconductors and insulators. In these solids, the transport of hot electrons has been extensively studied, both theoretically and experimentally, due to its importance in correctly modelling the transport of carriers in semiconductor devices [18].

\section{PARTICLE APPROACH}

The particle approach to describe dynamics within an irradiated sample can be applied to classical systems. It solves classical equations of motion of all involved particles. Scattering events may occur while the particles travel within the medium. Their rates are estimated a priori with dedicated theoretical approaches. The particle approach can be deterministic: molecular dynamics (MD) [5, 6] or stochastic: Monte Carlo (MC) [4]. The latter can be computationally more efficient for complex stochastic systems. In our application it 


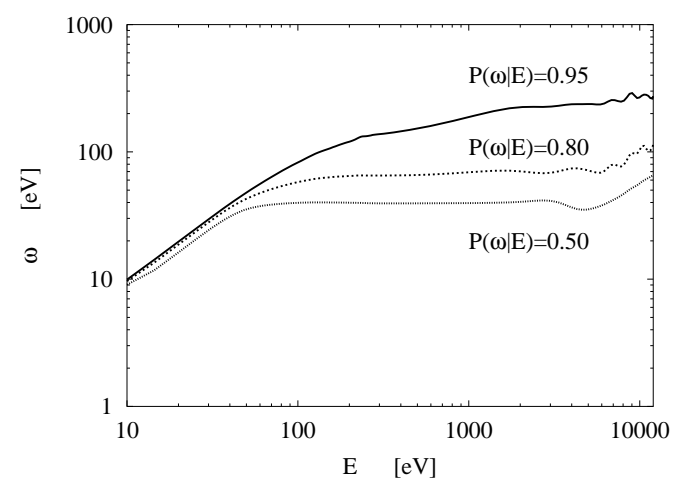

FIG. 1: Energy loss, $\omega$, in a single inelastic scattering event during electron-atom interactions in diamond as a function of electron energy. Plots show results at fixed integrated probabilities, $P(\omega \mid E)=0.5,0.8,0.95$, obtained with the TPP-2 model. The probability $P(\omega \mid E)$ denotes the integrated probability that the energy loss in a scattering event is less than or equal $\omega$.

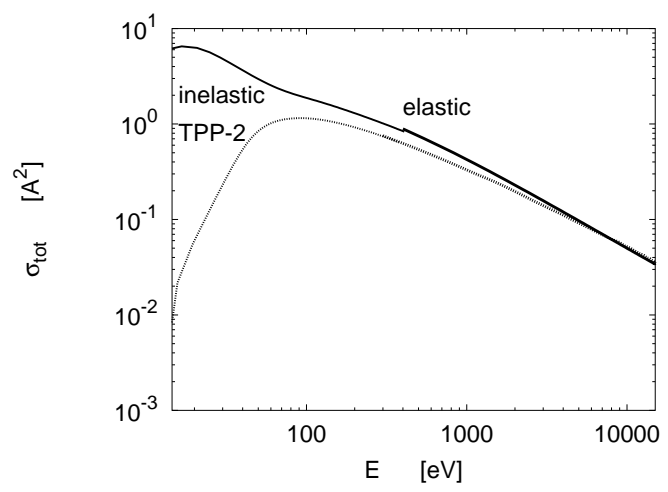

FIG. 2: Elastic and inelastic total cross sections for diamond. Inelastic cross sections are obtained from the Lindhard approximation with core ionization taken into account, using the TPP-2 optical model. Elastic cross sections (up to energies, $E=0.4 \mathrm{keV}$ ) were derived with the Barbieri/Van Hove Phase Shift package [19]. For larger energies, the elastic cross sections were taken from the NIST database.

applies particularly well when describing time evolution of an electron distribution. Below we discuss the details of MC method for semiconductors and metals, using two specific examples: diamond and aluminium. 


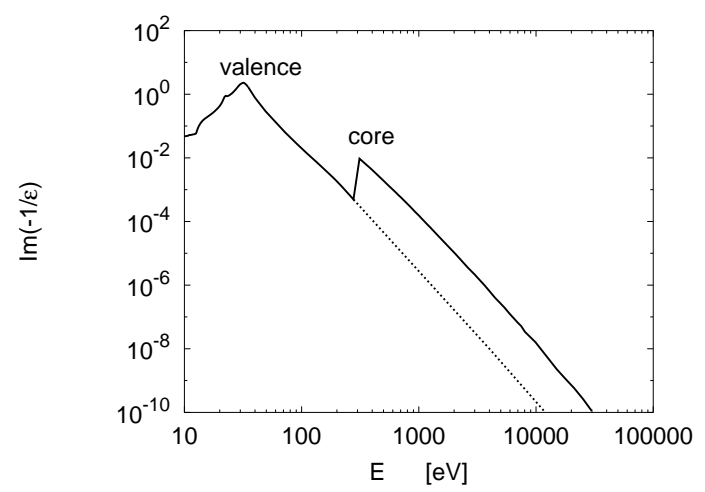

FIG. 3: Energy loss function of diamond, $\operatorname{Im}(-1 / \epsilon)$ : experimental estimate.

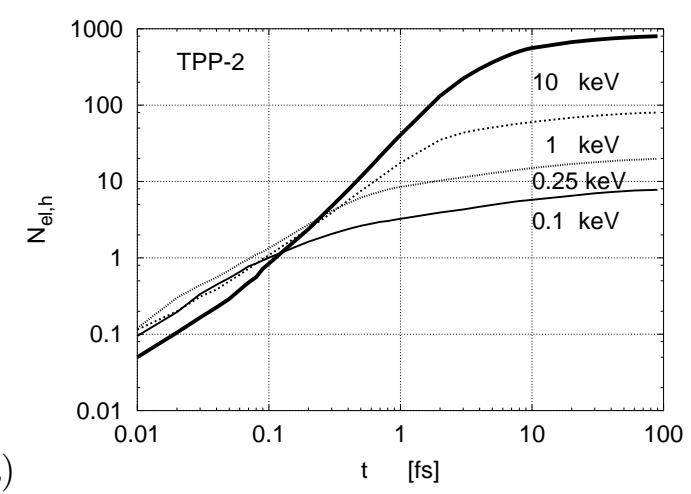

b)

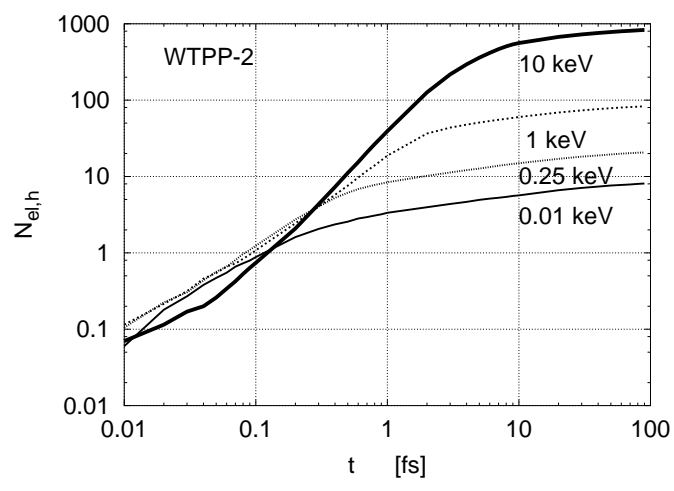

FIG. 4: Average number of secondary electrons or holes produced, $N_{e l}=N_{h}$, vs. time. Curves correspond to the results obtained at different electron impact energies $E=0.1-10 \mathrm{keV}$ with (a) the TPP-2 model, (b) the WTPP-2 model.

\section{A. Modelling electron cascades in irradiated diamond with a dedicated Monte-} Carlo code

During the simulation the MC code has to solve separate equations of motion for each particle in the sample, following its trajectory and its interaction with other particles or external fields. If any scatterings occur, the scattering probabilities are estimated with quantum mechanical cross sections. Coordinates and velocities of particles are updated at each time step. Simulations of single events are repeated many times. Estimates of physical observables are obtained by averaging their values obtained from single events over the total number of events. Therefore these estimates have statistical error.

In the Monte-Carlo method [20-22] dedicated to describe the propagation of electrons 

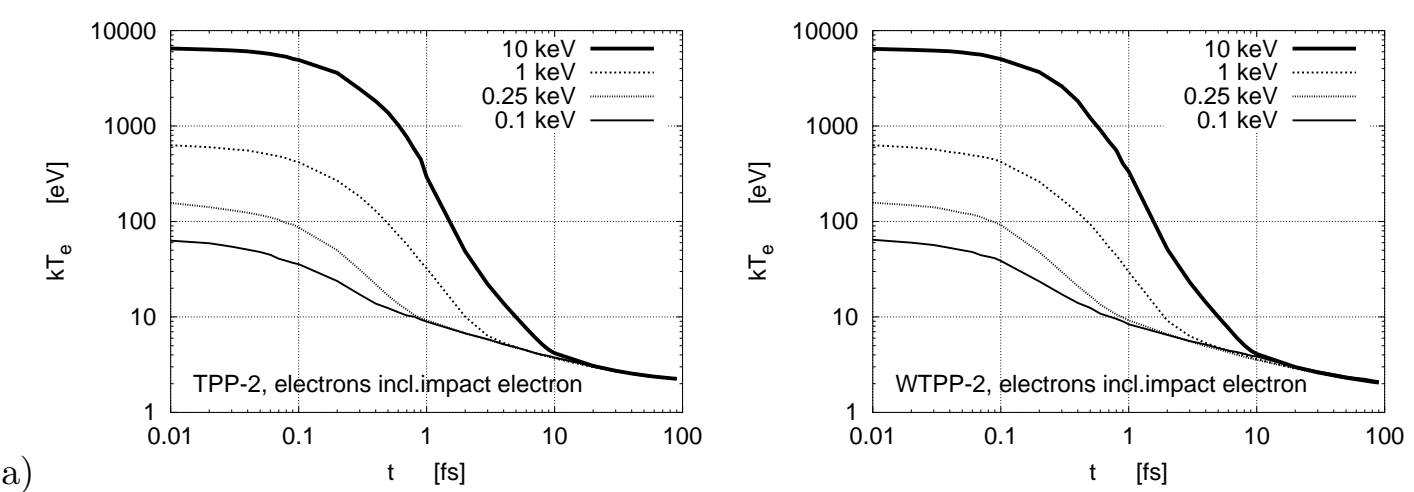

a)

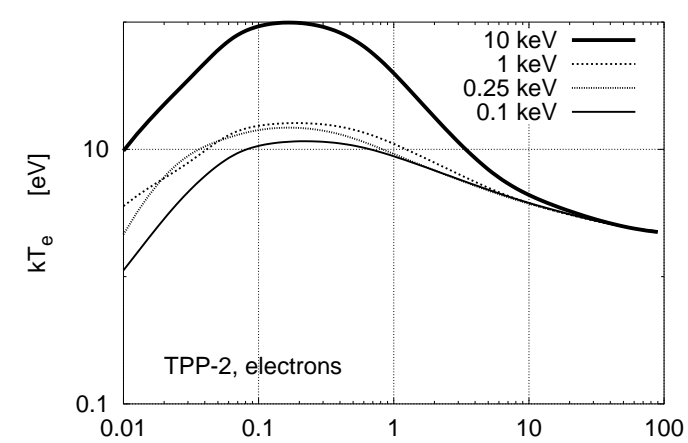

b)
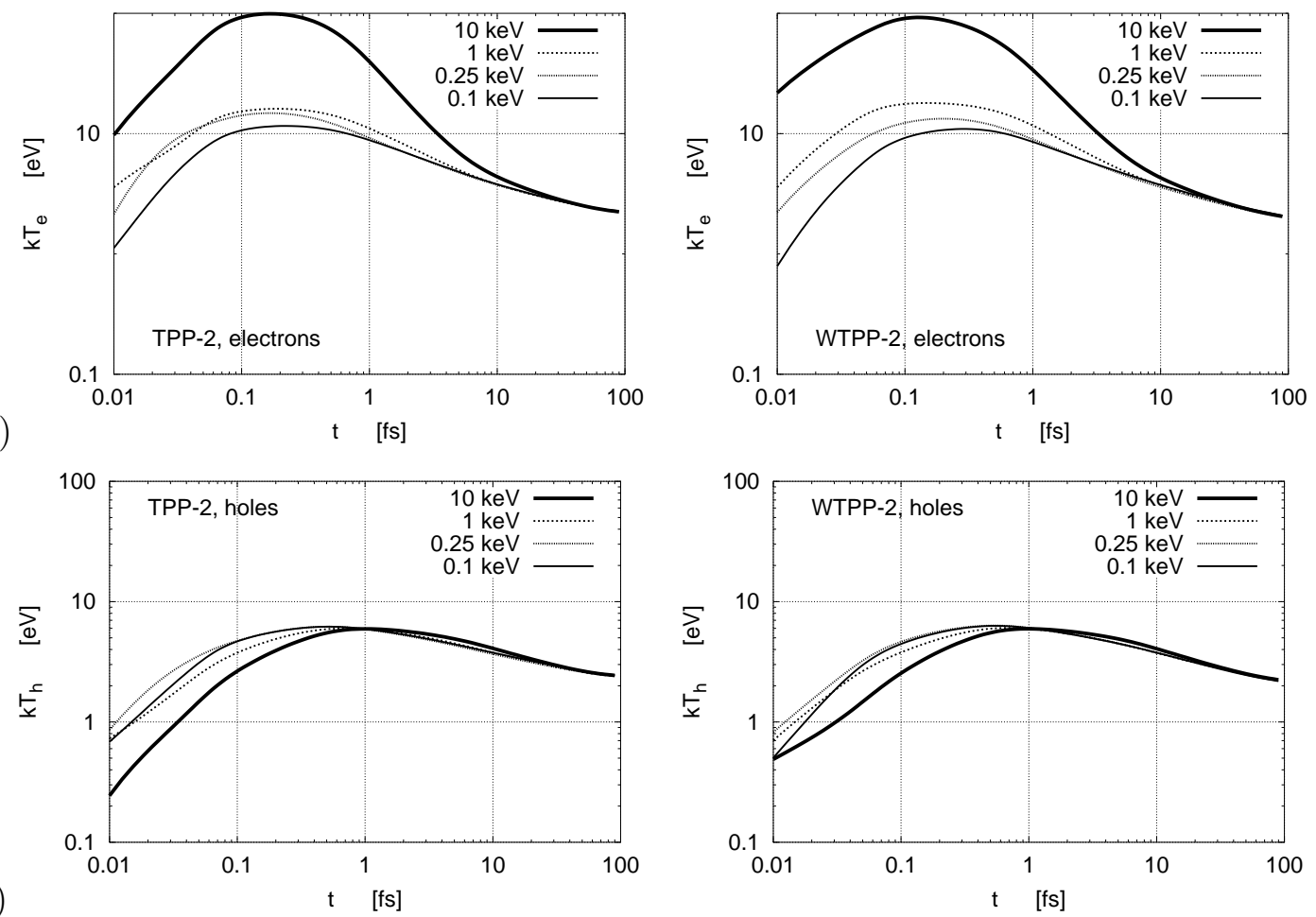

FIG. 5: (a-c) The kinetic temperature $k T$ of the carrier gas vs. time, averaged over 200 cascades. The temperature was calculated for (a) all electrons including the primary electron, (b) secondary electrons only, and (c) holes. Curves correspond to the results obtained at different electron impact energies $E=0.1-10 \mathrm{keV}$ from the TPP-2 model (left) and the WTPP-2 model (right).

in a solid, the moving electrons interact with the atoms of the solid. If the kinetic energy of the electrons is high, they propagate almost freely through the sample, and collide with single atoms $[23,24]$. This interaction may be either elastic or inelastic. Electrons also interact with the crystal lattice, emitting (and absorbing) phonons. These interactions may be neglected at high energies. Inelastic scattering usually results in an impact ionization followed by a release of an electron-hole pair in semiconductors and insulators. Simple 


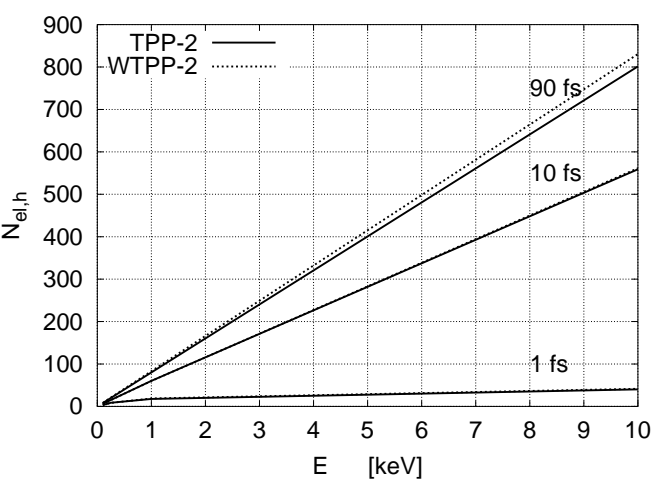

a)

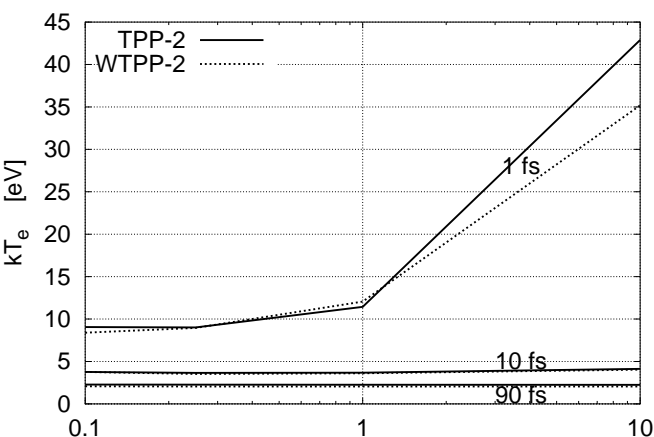

b)

$\mathrm{E} \quad[\mathrm{keV}]$

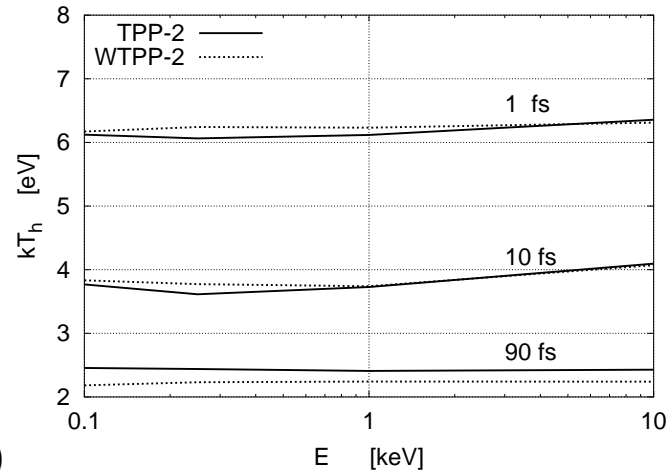

FIG. 6: (a) Average number of secondary electrons or holes emitted, $N_{e l}=N_{h}$, is plotted vs. energy, $E$; (b-c) The kinetic temperature $k T$ of the carrier gas, averaged over 200 cascades is plotted vs. energy, E. Curves correspond to the results obtained for: (b) secondary electrons, and (c) holes at different times $t=1,10,90$ fs from the TPP-2 model (solid line) and the WTPP-2 model (dashed line). The data in (a), (b), and (c) were sampled at primary energies of $E=0.1,0.25,1,10$ $\mathrm{keV}$.

scaling considerations predict that if valence electrons are ionized, their kinetic energies should not be much larger than a few times the shell binding energy. At higher impact energies the core electrons may also be ionized. Using the cross sections described below, Fig. 1 shows that the mean energy of the secondary electrons in diamond is about $40-60$ 


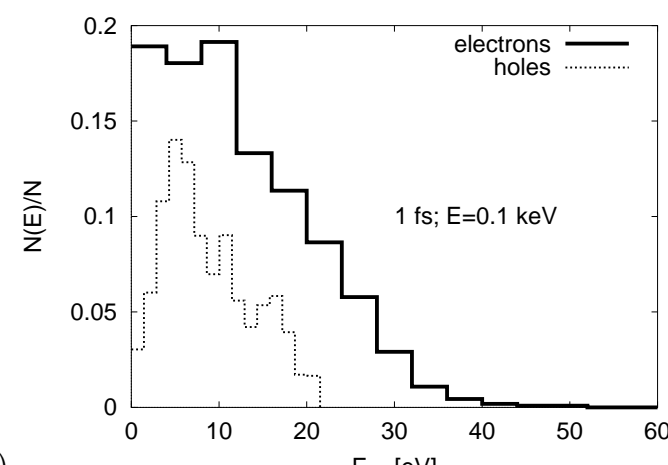

a)

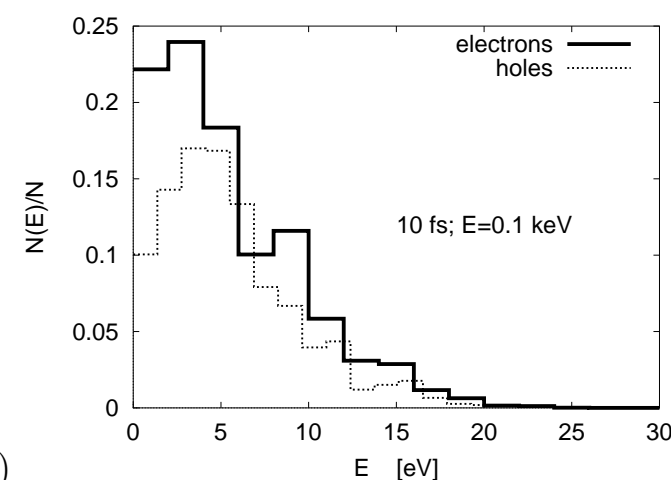

c)

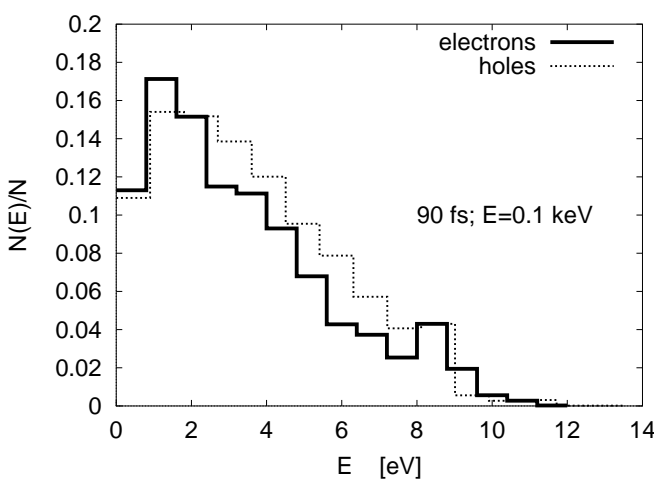

b)

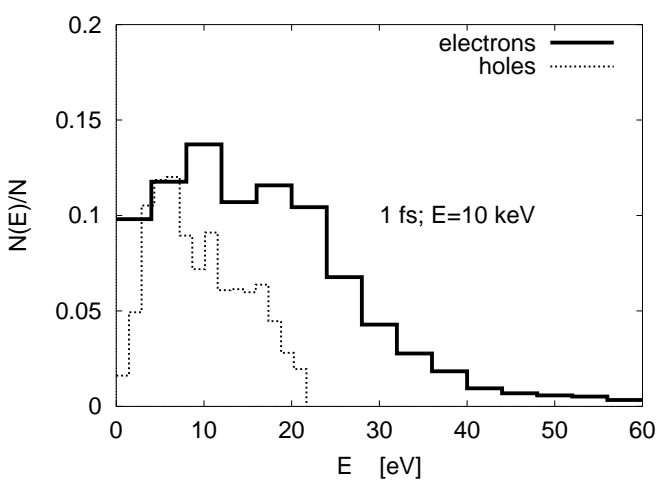

d)
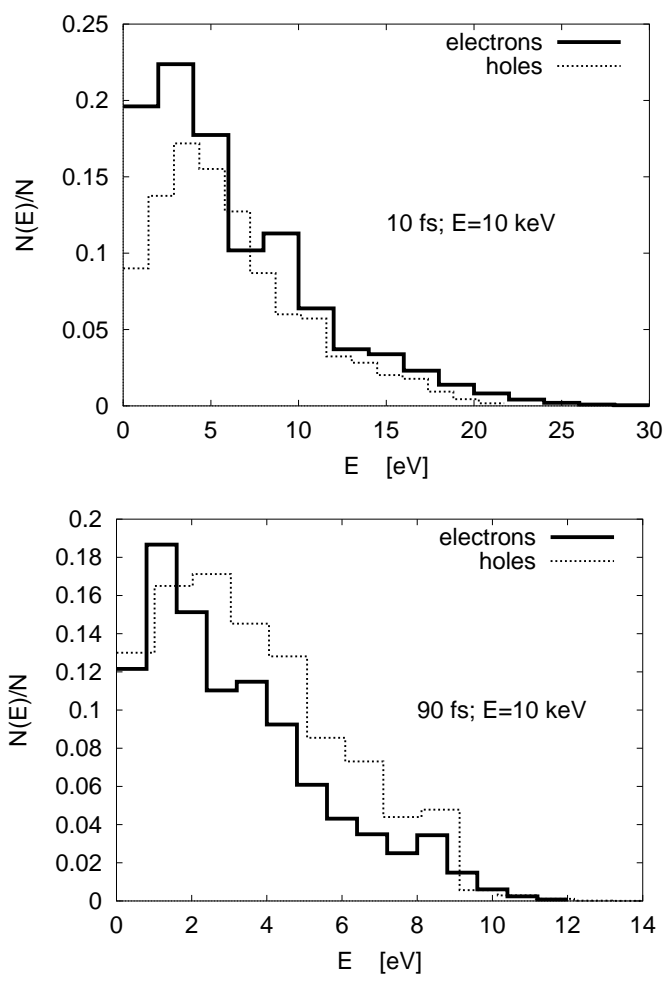

FIG. 7: Energy distribution, $N(E) / N$, (fraction of carriers per bin) among carriers at (a-b) $t=1$ fs; (c-d) $t=10$ fs; and (e-f) $t=90$ fs. Histograms correspond to results obtained at electron impact of $E=0.1 \mathrm{keV}$ (left) and $E=10 \mathrm{keV}$ (right) from the TPP-2 model for electrons and holes. The results are plotted for both electrons (solid line) and holes (dashed line).

$\mathrm{eV}$ (close to the energy of L shell electrons), reaching peak energies of about $300 \mathrm{eV}$ (close to the energy of core electrons). The energy loss, $\omega$, does not exceed $300 \mathrm{eV}$ (at the $95 \%$ probability level), and its value becomes independent of the impact energy, $E$, at energies greater than $1 \mathrm{keV}$. This can be expected as one-electron excitations are predominant in inelastic scattering $[25,26]$. 


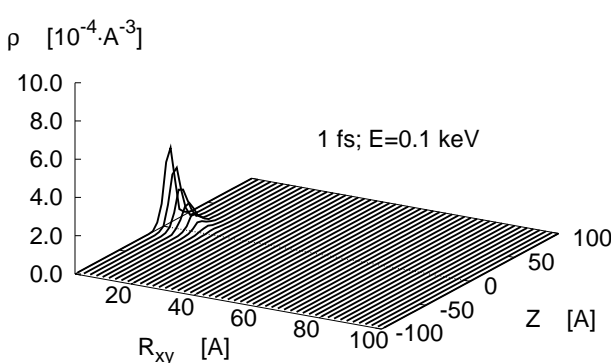

a)

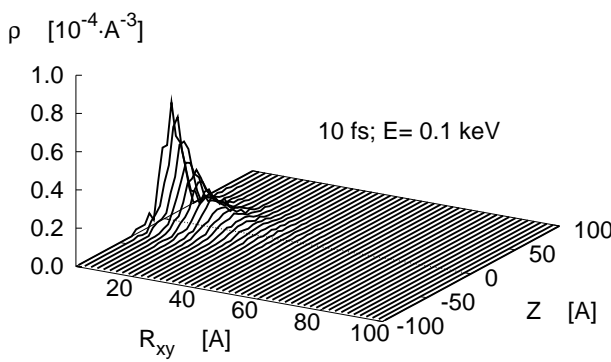

c)

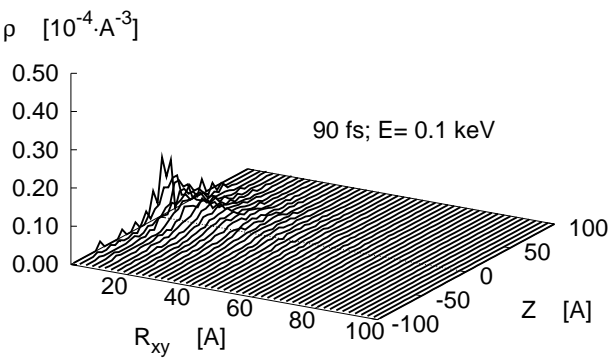

e)

FIG. 8: Local electron density estimated for the electron cloud. The data were collected at times: (a-b) $t=1 \mathrm{fs},(\mathbf{c}-\mathbf{d}) t=10 \mathrm{fs}$, (e-f) $t=90 \mathrm{fs}$ at primary energies of $E=0.1 \mathrm{keV}$ (left) and 10 keV (right) with the TPP-2 model. The data from the WTPP-2 model are similar (not shown).

\section{Calculations of electron trajectories}

When the incoming electron is fast, we can use the Bethe-Fermi approximation $[25,26]$. It replaces the electric field of the incoming electron by an electromagnetic pulse of the same short duration. Impact ionization is then proportional to the dipole transition probability caused by the short, non-periodic electromagnetic pulse. The Bethe-Fermi approximation is the basis of the optical model that we use below.

The primary (impact) electron loses its energy in a secondary electron cascade. When 
reaching an energy of $200-300 \mathrm{eV}$, the electron de Broglie wavelength becomes comparable with the atomic separation. At lower energies the electron interacts multiply with a cluster of neighbouring atoms, the size of which is of the order of the de Broglie wavelength [27]. For such low energies the Born approximation is not valid, and including a non-local exchange and correlation term into the interaction potential becomes necessary for an accurate description of electron scattering [28]. At the same time the electron-phonon coupling also increases although it is still small compared to the atomic electron-atom elastic cross section [29].

In this study, as in the previous ones [20, 21], we use the formalism of the Lindhard dielectric function [30] with the TPP-2 optical model [31-33] for the description of the inelastic interactions of electrons with atoms of the sample. This approach is based on the Bethe-Fermi approximation, and it takes into account both valence and core ionizations of the atoms in a solid, where the cross section for core ionizations is not higher than about 10 $\%$ of the total ionization cross section in the solid [34]. We use this model as it accurately provides the electron inelastic cross sections in solids over a wide energy range, $0.05<E<10$ $\mathrm{keV}$, particularly at lower energies $(E<200-300 \mathrm{eV})$, where the atomic models fail.

At low energies $(E<50 \mathrm{eV})$ inelastic cross sections calculated with the optical models cannot be fully trusted [28]. Exchange and correlation terms in the atomic potential, and the complex structure of the energy band strongly influence the dynamics of the scattered electrons, and an accurate calculation of the impact ionization rate should include these effects. The first-principles calculations are then required in order to estimate the ionization rate by electrons and holes. For diamond such calculations were performed by Watanabe et al. [35] at very low impact energies. In this approach the band structure was calculated using the empirical pseudopotential method, and the dielectric function was evaluated with the calculated band structure.

As in previous studies, we treat elastic scattering of the incoming electron with atoms in the muffin-tin potential approximation [20, 21, 23, 24]. To estimate the elastic scattering of low-energy electrons $(E<0.4 \mathrm{keV})$ we use programs from the Barbieri/Van Hove Phase Shift package [19]. For $E \geq 0.4 \mathrm{keV}$ we obtain elastic cross sections from the NIST database [36]. Figure 2 shows the total elastic and inelastic cross sections obtained from the calculations. The results show that for energies higher than $1 \mathrm{keV}$, the elastic and inelastic cross sections are comparable, but for lower energies, $0.1<E<1 \mathrm{keV}$, the elastic cross section 
is twice as large as the inelastic one. For very low energies, $E<0.1 \mathrm{keV}$, the inelastic cross sections drop rapidly, and elastic interactions become predominant. The electron-phonon coupling becomes significant for carriers of very low impact energies, $E<E_{G}[18,29,37,38]$.

\section{Core ionization}

Figure 3 shows the experimental estimate of the energy loss function (ELF) for diamond. The energy loss function is the sum of the large valence and small core contributions. We make a rough estimate of the pure core contribution by subtracting the valence component from the ELF. The valence component was extrapolated above the core ionization edge (cf. Fig. 3). The respective cross section for the core ionization can be obtained from ELF with the Lindhard approximation, using optical models. However, we neglect the relaxation processes (Auger decay) following a core hole formation.

\section{Holes}

Impact ionization of a valence electron in a semiconductor or an insulator always releases a pair of carriers: an electron and a hole. When far from the solid surface, the holes behave as free carriers moving inside the valence band. A dispersion relation depending on the band structure describes the relation between the energy and the momentum of the hole. The holes may cause further impact ionizations, and the respective ionization cross sections can be estimated from first-principles calculations with the band structure [39-43]. The results obtained for several semiconductors [18] show that these cross sections are comparable to the cross sections for ionization by impact electrons. This approach may be also applied for diamond where the effective mass of a hole is similar to the mass of a free electron [44]. Therefore we use the electronic impact cross sections as a first-order approximation for the ionization cross sections by a hole impact.

\section{Impact ionization at very low energies}

In semiconductors, the rate of impact ionization at low energies based on first-principles calculations can be fit as, $\Gamma=a\left(E-E_{t h}\right)^{b}$, where $a$ and $b$ are coefficients specific for the semiconductor, and the threshold energy, $E_{t h} \geq E_{G}$ [40-43]. Coefficients $a$ and $b$ are calculated to get $\Gamma$ in $[1 / \mathrm{fs}]$ units with energy expressed in $[\mathrm{eV}]$ units. We fit this relation to the ionization rate obtained by Watanabe et al. [35] for diamond with $a=5.53 \cdot 10^{-5}$, 
$b=4.33$ and hence obtained the respective mean free path, $\lambda=v_{e, h} / \Gamma$, where $v_{e, h}$ denoted the velocity of the carrier. The energy of the primary electron after ionization and the energies of secondaries were obtained within the random-k approximation $[45,46]$.

\section{Band structure}

Calculating the conditional probabilites, we used the densities of states of the valence and the conduction bands obtained from the band structure calculations of Barnard, Russo and Snook [47]. These calculations were performed for crystal diamond. The (unnormalized) densities of states were evaluated with the CRYSTAL98 code for both the valence and the conduction band.

\section{Momentum conservation}

After impact ionization at high impact energies the differential cross sections determine the energy loss of the primary electron and its scattering angle. At low impact energies we can obtain the energies of all carriers: the primary electron, the secondary electron and the hole with the random-k approximation $[45,46]$. The scattering angle of the primary carrier is then chosen randomly. The energies of carriers are proportional to the density of states (DOS) of the valence band in which they are created. The energies of the primary and secondary carriers determine the magnitude of their momenta, following the dispersion relations in the respective bands. Here we assume the quadratic dispersion relation for both electrons and holes [27].

The momentum transfer, $\Delta \mathbf{k}=\mathbf{k}_{i}^{\prime}-\mathbf{k}_{i}$, is then estimated from the scattering angle of the primary carrier, and the momentum conservation for the production of an electron-hole pair by the carrier requires,

$$
\mathbf{k}_{e}^{\prime}+\mathbf{k}_{h}^{\prime}=-\Delta \mathbf{k}
$$

where $\mathbf{k}_{e}^{\prime}, \mathbf{k}_{h}^{\prime}$ denote the momenta of the electron and hole released.

\section{Phonons}

The accurate calculation of the carrier-phonon scattering in a semiconductor requires complex first-principles calculations including the band structure [18]. However, the energy gains or losses due to phonons are small, $E<0.1 \mathrm{eV}$, and do not influence significantly the dynamics of impact ionization. Since we are primarily interested in processes that con- 
tribute significantly to the impact ionization rate, we did not take these gains and losses into account.

The carrier-phonon scatterings that occur mostly at low energies will then only lower the ionization rate of the sample. Therefore, we do not require high accuracy in the description of the phonon coupling, and we describe this coupling in our model, using a simple fit. We assume that at very low energies the carrier-phonon total scattering rate is proportional to the density of states, $\sim \rho_{V(C)}(E)[40,48]$, and that at higher energies this rate decreases as $\sim 1 / \sqrt{E}[29]$. These two approximations are linked at the energy of about $5 \mathrm{eV}$ which corresponds to the deformation potential constant calculated for acoustic phonon in diamond [49]. The total scattering rate thus obtained was used to estimate the mean free paths for the electron- and the hole-phonon scattering in diamond. However, possible gains and losses of carrier energy occurring in these scatterings are neglected in the simulations.

\section{Results on impact ionization by electrons and holes}

Using the model described above, we performed a set of Monte Carlo simulations to follow the path of an impact electron and of the secondary electrons and holes in diamond. We used a coordinate system with the starting position of the impact electron (at $t=0$ fs) at the origin, and the velocity of the impact electron along the Z-axis. The space-time characteristics of secondary cascades of electrons and holes were recorded as a function of the impact energy.

\section{Evolution}

The evolution of each cascade was analysed through the number of secondary electrons, $N_{e l}(t)$, and the kinetic temperature of the free electron gas $k T(t)=2 / 3 \cdot E / N$, where $E$ is the total kinetic energy of electrons, and $N=N_{e l}+1$ is the total number of electrons. These quantities were averaged over 200 cascades. Figures 4, 5 show the results obtained with the TPP-2 optical model and the WTPP-2 model for impact energies of $E=0.1,0.25,1,10 \mathrm{keV}$.

The number of electrons emitted increased in time, and saturated within 10-100 fs with a total of about $8,20,80$ and 800 electrons released at impact energies of $E=0.1,0.25,1,10$ $\mathrm{keV}$ respectively.

In the context of nuclear radiation detectors, several experimental studies have found an 
average pair creation energy by high energy electrons and ions in diamond between 12.8 and $13.6 \mathrm{eV}$ [50-52] with $13 \mathrm{eV}$ being the most recent result [52]. Previous theoretical work on cascades initiated by electrons suggests pair creation values between 10.3 and $11.6 \mathrm{eV}$ $[53,54]$. Experimental values for the pair creation energy agree well with the values found in our simulations, $12.5 \mathrm{eV}$ with the TPP-2 model, and $12 \mathrm{eV}$ with the WTPP-2 model. For example, using $13 \mathrm{eV}$ as the average pair creation energy, one would predict about 770 ionizations for a $10 \mathrm{keV}$ electron, compared to values of 800 and 830 obtained with the TPP-2 and WTPP-2 models respectively.

While simulating the cascades of secondary electrons and holes initiated by single electron impacts, we used the elastic and inelastic cross sections calculated for a neutral solid. That was a good approximation as in that case the fraction of ionized atoms in the sample was small. If carrier cascades are initiated by many impact electrons simultaneously, the fraction of ions in the sample becomes large, and the approximation of a neutral medium breaks down. However, the cross sections for the impact ionization of the ions are much smaller than the cross sections for the impact ionization of neutral atoms. Therefore our results obtained within the neutral medium approximation represent the upper limit of the number of possible impact ionizations occurring in a real case, when a fraction of atoms has been ionized.

The kinetic temperature of the electron gas (calculated including the primary electron) decreased as the cascade evolved (Fig. 5a). We note that after about $10 \mathrm{fs}$, all temperature curves showed similar overall features, and these were independent from the energy of the primary impact particle. This indicated efficient electron thermalization during the cascading process within the sample. At 100 fs the temperature of the electron gas dropped to $\sim 2-2.2 \mathrm{eV}$. In a similar way we estimate also the kinetic temperature of the secondary electrons and holes shown in Figs. 5b-c. The results show similar curve shapes for secondary electrons and holes. The kinetic temperature of the carrier gas increased to a peak value within $\sim 0.1$ fs (electrons) and $\sim 1$ fs (holes), and then decreased, reaching (at $\sim 10 \mathrm{fs}$ ) a final temperature independent of the primary impact energy.

Figures 6a show plots of the average number of electrons released, $N_{e l}$; (b-c) the kinetic temperature, $k T$, of the carrier gas as a function of the energy, $E$, of the primary electron at different times. These curves describe results obtained at 1,10,90 fs, and were based on the TPP-2 model and the WTPP-2 model. The data represent primary energies of 
$E=0.1,0.25,1,10 \mathrm{keV}$. The results can be used for the interpolation of the number of ionizations, and the temperature of the electrons at energies ranging from $E=0.1 \mathrm{keV}$ to $E=10 \mathrm{keV}$. The results show that the number of secondary electrons, $N_{e l}$, is proportional to the impact energy.

\section{The energy distribution of secondary electrons}

The positions and velocities of electrons recorded at times, $t=1,10,90 \mathrm{fs}$ at energies of $E=0.1,0.25,1,10 \mathrm{keV}$ were collected from all cascades. Histograms for the energy distributions were obtained, $N(E) / N$, at these time points. Number, $N(E)=\sum_{i=1}^{200} N_{i}(E)$, is the total number of electrons in a bin, $(E, E+\Delta E)$, obtained from summing up the results, $N_{i}(E)$, from all cascades. Correspondingly, $N_{i}(E)$ is the number of electrons found in that bin for the $i$ th cascade. These distributions were normalized to the total number of electrons, $N=\sum_{E} N(E)$. Fig. 7 shows the histograms at impact energies of $E=0.1$ $\mathrm{keV}$ and $E=10 \mathrm{keV}$. As expected, the energy histograms show that the number of lowenergy electrons increased with time. One may notice that the dissipation of the impact energy is fast. Both the TPP-2 and the WTPP-2 models predict that at $t=1$ fs after the primary emission most of the electrons will have energy lower than $60 \mathrm{eV}$, independently of the energy of the primary impact. Similarly, at 10 fs the energy of the most of the electrons will be lower than $25 \mathrm{eV}$, and at 90 fs lower than $12 \mathrm{eV}$.

The plots show the characteristic and well-known shape of the secondary electron (hole) distributions $[55,56]$ : the distribution rapidly increases at very low energies, reaching a peak value at a few electron volts, and then smoothly decreases, forming a long tail extending to high energies. The position of the peak moves towards lower energies, as the number of secondary electrons increases. The peak is located at $\sim 5 \mathrm{eV}$ after 10 fs from the primary electron emission, and at $\sim 2 \mathrm{eV}$ after $100 \mathrm{fs}$. The distributions of hole energies show a similar tendency. The histograms for electrons and holes become comparable at $t=90$ fs after the primary electron emission, when the impact energy has already dissipated. 


\section{Spatial distribution of secondary electrons}

In order to describe the spatial distribution of the secondary electron cloud in cascades triggered by $E=0.1$ and $E=10 \mathrm{keV}$ electrons, results from all simulations were analysed at these energies. Figure 8 shows the local normalized density of electrons and holes from the TPP-2 model at times, $t=1,10,90$ fs created by single electron impacts with velocity along the Z-axis. The normalization condition requires, $\sum_{i} \rho_{i} V_{i}=1$, where $\rho_{i}$ is the carrier density measured in the volume, $V_{i}$. The (normalized) density of electrons is distributed on the $R_{x y}=\sqrt{x^{2}+y^{2}}$ and $Z$ plane and localized around the position of the primary impact. At longer time scales, $t>10 \mathrm{fs}$, the density becomes smoother, and this peak becomes smaller. At 90 fs the electron cloud spreads to $\left(R_{x y}, Z_{-}, Z_{+}\right)=(60,-100,70)$ Angstroms and $\left(R_{x y}, Z_{-}, Z_{+}\right)=(200,-200,800)$ Angstroms at energies $E=0.1$ and 10 $\mathrm{keV}$ respectively. Results from the WTPP-2 model were very similar and are not shown separately.

\section{Accomplishments of the model}

This simple independent-electron-approximation model was constructed to describe the dynamics of the impact ionizations triggered by an electron or a hole impact in diamond. The model combines a Bethe-Fermi treatment, using optical model approach at high energies, with a band structure model at low energies. Impact ionization by holes was included. The results obtained have been used to estimate ionization by electrons of different impact energies $(E=0.1-10 \mathrm{keV})$ in diamond. The main results are: (i) 8-800 secondary electrons released at different impact energies $E=0.1-10 \mathrm{keV}$. These numbers are in agreement with previous experiments and calculations, (ii) time dependent results, showing that the typical cascade time ranges from 10 fs to 100 fs at $E=0.1-10 \mathrm{keV}$. The spatial extent of the electron cloud ranges from $\left(R_{x y}, Z_{-}, Z_{+}\right)=(60,-100,70)$ Angstroms at $E=0.1 \mathrm{keV}$ to $\left(R_{x y}, Z_{-}, Z_{+}\right)=(200,-200,800)$ Angstroms at $E=10 \mathrm{keV}$. The energy distribution is asymmetric. It peaks at low energies and then smoothly decreases, forming a tail at higher energies.

The Monte-Carlo code may be adapted to simulate ionization phenomena in different systems, ranging from the explosion of atomic clusters to the formation of warm dense matter and plasmas. The model can also be used to estimate ionization rates and the 
spatio-temporal characteristics of secondary electron cascades in biological substances.

For $10 \mathrm{keV}$ X-rays, as generated by XFELs, the electron cascade will develop within 100 fs after the primary photoelectron emission. Therefore the dynamics of the electron cascade is quite important in the interaction of XFEL beams with materials such as diamond. It may be possible to perform time-resolved studies of such electron cascades with XFELs.

\section{B. Monte-Carlo code to follow electron dynamics in irradiated aluminium}

In the previous section we have presented the Monte Carlo method working in the framework of the independent electron approximation. This approximation limits the applicability of the method to low laser fluences, or to higher electron energies, because otherwise the correlation among electrons cannot be neglected. In this section we will demonstrate how the electron correlations can be treated within the MC approach. This is especially important for the case of correlated many-electron systems. For electrons of lower energies, i.e., from a few tens up to a few hundreds of $\mathrm{eV}$, and at high fluences, which are typical for the new generation of XUV femtosecond lasers (FLASH), the previous MC concept is not applicable directly. However, some modifications of the method allow its use in the correlated regime [57-59].

As discussed in the previous section, the simulation method must take into account the density of states of the material, as well as all dominant electron scattering channels, including core ionization and the ensuing relaxation processes. Additionally, the inclusion of Pauli's principle is essential to maintain the Fermi-distribution of electrons, instead of the classical Maxwell-Boltzmann distribution. This will be discussed in more detail later.

Here we present a Monte Carlo method, applied to model the effect of a 10 fs Gaussian laser pulse on a bulk of solid aluminium [57-59]. For each arriving radiation photon its realized free path is calculated from Poisson's law. The mean free path is taken from Ref. [60]. We assume that a radiation photon is absorbed, if its realized penetration depth is smaller than the size of the simulation box. For modeling we use a simulation box of $10 \times 10 \times 10 \mathrm{~nm}^{3}$ size, which is much smaller than the typical laser spot $(\sim 1 \mu \mathrm{m}$ to $10 \mu \mathrm{m})$. Therefore, we use periodic boundary conditions.

The electron that absorbs a photon is chosen randomly among all the electrons available within atomic shells or within the conduction band, with the probability weights given by 
the probabilities of photoabsorption by different shells and the specific DOS in Al. Note that for photons of energy above the L-shell ionization potential, the probability of photoabsorption by electrons from L-shell is much higher than by the conduction band electrons [60].

\section{Individual particles}

The solid aluminium has a density of $n_{a t}=5.4 \times 10^{28} \mathrm{~m}^{-3}$ and is considered as an isotropic and homogeneous medium, which allows us to use a Poisson distribution to estimate the realized free path between sequential collisions. The inelastic/elastic mean free path is calculated from the corresponding cross-sections. In contrast to semiconductors, there is no valence band in a metal. All conduction band electrons in the simulation box are treated as individual particles within the MC procedure. The initial electron distribution within the conduction band of $\mathrm{Al}$ fills the energy levels up to the Fermi energy, following the DOS of the material, Refs. [61, 62]. All the electrons of energy higher than the 'outermost' state of the conduction band are treated as free electrons, i.e., as electrons with a free-electron DOS. All electronic effective masses are chosen to be equal to the free electron mass.

The estimates for the electron scattering cross sections were discussed in the previous subsection. Here, we apply a cross-section for impact ionization of bound electrons in a simple analytical form proposed by Gryzinski [63]. Another alternative is the cross-section given by Lotz in Refs. [64, 65]. Further, one could use a more complex dielectric function formalism to account for collective effects in solids [66]. The interaction between two free electrons is described by a dynamically screened Coulomb potential within the Lindhard dielectric formalism, equivalent to the Lenard-Balescu equation [66, 67]. In order to describe this interaction, one can also apply various models: the Mermin dielectric function, or the Full-Conserving-Dielectric-Function [67]. But as shown in Ref. [67], the Lindhard dielectric function remains in good agreement with these more complex approaches.

For the description of the Auger processes, filling holes in the L-shell, we apply an exponential law for the time of decay, with a material-dependent time constant $[66,68]$. The electrons involved in the Auger transition as well as their partners for free-free collisions are randomly chosen among the electrons in the conduction band. The characteristic time of L-shell Auger decay for aluminium is $\sim 40 \mathrm{fs}$ [69].

While modeling the secondary ionization, we consider an electron to be ionized, if the transferred energy is higher than the ionization potential, which is at $72 \mathrm{eV}$ below the Fermi 
energy $E_{F}$ for L-shell of $\mathrm{Al}$, and if there are vacancies available. Subsequent propagation of secondary electrons and their interactions with the target are also taken into account.

After a realized event, the particle changes its state: (i) a photon disappears creating a new free electron, (ii) a hole moves up or also disappears, exciting an electron, and (iii) a colliding electron changes its energy, and may change the energy of a second electron in case of inelastic or free-free scattering. Afterwards, the paths for the remaining particles are calculated. The secondary particles are traced with the same algorithm. In this way, we achieve the time-resolved accounting of all particles within our simulation box.

\section{Distribution functions}

Since every change of an electronic state changes the electron distribution function, we also take this into account in our simulation. This is important because: (i) Pauli's exclusion principle is imposed on the transient distributions, which are changing with time, and (ii) the correlation effects must be considered for the case of metals [59]. Indeed, after appearance of a "hole" within the conduction band below the Fermi-energy, any of the free electrons can interact with any other to fill this hole. A hole is formed, when a highly energetic free electron interacts with a low energy electron from the bottom of conduction band (below the Fermi-energy), or when an electron is removed from the low energy state by photoabsorption or Auger decay. The system then rearranges its state so that all electrons should have a chance to interact with the hole and not only the highly excited ones. To enable this, we use discretized time intervals of $0.5 \mathrm{fs}$, which are short, compared to the typical timescales of the problem. At each time step we redistribute the paths of the electrons, which did not interact before, giving them now a chance to interact. In this approximate way we include possible time correlations between electrons.

Our algorithm simultaneously tracks all individual particles, and the discretized electron distribution function, $f_{c}(E)$, on a grid. The index, $c$, denotes the conduction band electrons, while for semiconductors the index $v$ would refer to the valence band [68]. At each collision, when an electron leaves an energy state $E_{1}$, the distribution of electrons changes as: $f_{c}\left(E_{1}\right) \rightarrow f_{c}\left(E_{1}\right)-1 / g\left(E_{1}\right)$, where $g\left(E_{1}\right)$ is the DOS of our target material, which corresponds to the loss of one electron from this energy state. At the same time, for an electron incoming to the energy interval $E_{2}$, the distribution function is increasing by one electron: $f_{c}\left(E_{2}\right) \rightarrow f_{c}\left(E_{2}\right)+1 / g\left(E_{2}\right)$. The same procedure must be repeated for the second electron 


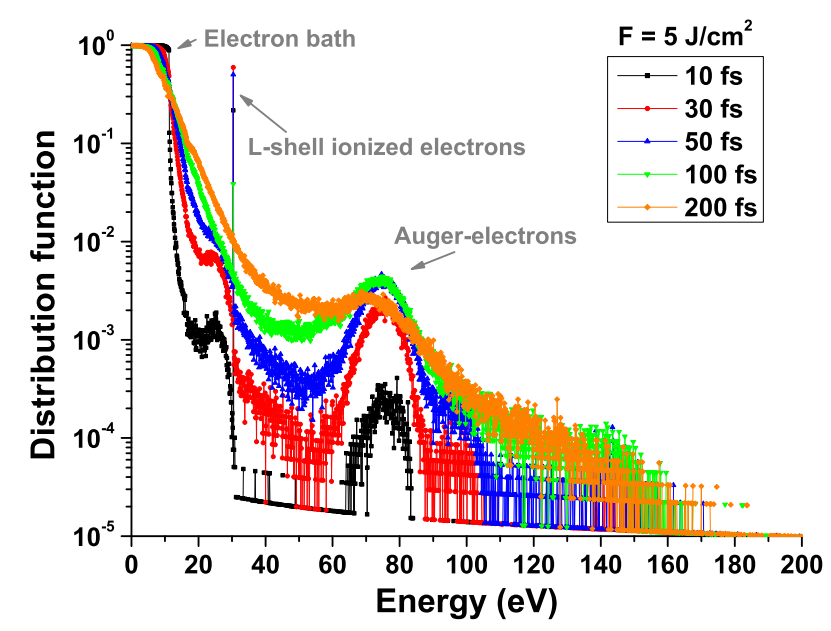

FIG. 9: (Color online). Electron distribution function at the fluence of $5 \mathrm{~J} / \mathrm{cm}^{2}$ and the photon energy of $92 \mathrm{eV}$ calculated at different times during and after the exposure.

involved in the interaction.

Pauli's principle forbids transitions of electrons into fully filled states, where $f\left(E_{i}\right)=$ 1. That is usually accounted for by multiplying every collision probability with a Pauli factor, $F=\left(1-f_{c}(E)\right)$. In our simulation we introduced this factor into the mean free path $[59,68,70]$. Therefore, during the simulation only excitations into energy intervals, containing vacancies, are possible. In the case when there are no vacancies for electrons the mean free path of such collision calculated from the Pauli-principle-modified cross-section becomes infinite, and this event never realizes. Exactly this feature allows us to obtain the Fermi-distribution of electrons, instead of the classical Maxwell-Boltzmann distribution [59]. This extends the limits of applicability of the classical Monte Carlo scheme to lower energy electrons.

Also note, that if two electrons are involved in the interaction (impact ionization or free-free scattering), there must be two Pauli's factors applied, each responsible for the probability of a vacancy in the respective final state for each electron. 


\section{Accomplishments of the model}

With the MC code described above, we obtained the transient energy distribution of electrons within the conduction band. If we irradiate aluminium with photons above the Lshell ionization potential, corresponding to experimental parameters from [71, 72], a part of their energy will be stored in deep holes, in a manner similar to the case of semiconductors. But since the characteristic Auger time of L-shell decay in aluminium is $40 \mathrm{fs}$, the potential energy will be returned to the electrons fast, within approximately 200 fs, see Fig. 9.

In Fig. 9, the transient electron distribution function is plotted. It typically consists of several parts: (i) the bath of conduction band electrons which is close to Fermi distribution at a non-zero, time-dependent temperature, (ii) a photoexcitation peak at $30 \mathrm{eV}$ formed after the photoexcitation of L-shell electrons by $92 \mathrm{eV}$ photons, (iii) the high energy Auger electrons at the energies of $73 \mathrm{eV} \pm E_{F}$, and iv) the long energy tail for high fluences arising from photoabsorption of free electrons. The data are not smooth due to the Monte Carlo procedure applied here.

Without an external energy input, the electrons would quickly relax into a Fermi distribution function. However, the short-time dynamics here is driven directly by photoabsorption and indirectly via the Auger processes. After the excitation of an electron into the highenergy states, this electron redistributes its energy by interacting with other conduction band electrons. If it loses sufficient energy, it can thermalize with the Fermi electron bath, raising its temperature. However, this thermalization takes a relatively long time: as shown in Ref. [57] for the fluence of $5 \mathrm{~J} / \mathrm{cm}^{2}$, the distribution reaches a Fermi-like shape after approximately 400 fs.

Interestingly, a partial thermal equilibrium state of low energy electrons, with energies below $\sim 2 E_{F}$, appears already during or shortly after the x-ray pulse starts, while the high-energy part of the distribution remains nonthermal. This special form of the transient electron distribution, referred as the "bump on hot tail" in Ref. [73], occurs in materials irradiated by VUV and shorter-wavelength photons. The high-energy bump may originate directly from excitations of valence band in case of dielectrics, semiconductors, from atoms in a plasma, or from Auger decays, as in the present case. This specific shape of the transient electron distribution enables, in principle, the treatment of its high- and low-energy part with two different simulation methods. 
Excellent agreement of the spectra calculated from the simulated transient electron distributions with the experimental data [57] confirms the applicability of the method developed to describe the evolution of materials excited by XUV femtosecond lasers.

Finally, at these relatively low fluences that we considered here, the electronic structure is close to that of cold aluminium [71]. Thus, the ground state approach is still applicable. For higher fluences, changes in the electronic band structure and scattering rates must be taken into account. A possible way to account for such effects will be discussed at the end of this review.

\section{KINETIC APPROACH}

We now present a computationally efficient method of describing the evolution of electron density within irradiated large systems, which is applicable to solids.

This first-principles Boltzmann method is based on the statistical description of the charge dynamics in terms of statistical quantities: electron and ion densities in phase space, $\rho^{(e, i)}(\mathbf{r}, \mathbf{v}, t)$. These densities are functions of the spatial and velocity coordinates, $\mathbf{r}$ and $\mathbf{v}$, and are measured at time, $t$. The quantity, $\rho^{(e, i)}(\mathbf{r}, \mathbf{v}, t) d^{3} r d^{3} v$, estimates the number of particles (electrons or ions) in an volume element of phase space, $d V=d^{3} r d^{3} v$, which is located at the spatial point, $\mathbf{r}$, and at the velocity, v. Charge densities are evolved from their initial configuration at $t=0$, using semiclassical Boltzmann equations.

The Boltzmann method is a promising alternative to the first-principles Monte Carlo (MC) or Molecular Dynamics (MD) methods which are commonly used [10, 74, 75]. Monte Carlo algorithms have a transparent structure and usually do not require an application of any complex numerical methods. This is a great advantage of this method. However, these algorithms become computationally inefficient when the number of particles is large. The code efficiency is even worse, if the long-range interactions between particles, e.g., Coulomb electrostatic forces, have to be included into the simulation. High computational costs that scale with the number of particles restrict the applicability of the particle methods to the samples of small or moderate size.

In contrast, the efficiency of the simulation algorithm with the Boltzmann equations does not change directly with the number of particles in the sample, as the algorithm operates on smooth density functions. The efficiency and accuracy of these algorithms depend only 
on the phase-space shape of the sample that is reflected by the number of grid points used in the simulation. Therefore the statistical approach can also work well for large samples, where the MD/MC methods are inefficient.

The full spatio-temporal characteristics of the electron and ion dynamics can be easily obtained with the kinetic approach. As charge densities are directly evolved with Boltzmann equations, the averaged observables, $O$, of interest can then be calculated with their convolution with the charge densities obtained, $\langle O(t)\rangle=\int O(\mathbf{r}, \mathbf{v}) \rho(\mathbf{r}, \mathbf{v}, t) d^{3} r d^{3} v$. These results are not biased with statistical errors.

Below we write a general form of kinetic equations within an irradiated (atomic) sample [76]. The coupled semi-classical Boltzmann equations for single electron, $\rho^{(e)}(\mathbf{r}, \mathbf{v}, t)$, and ion densities, $\rho^{(i)}(\mathbf{r}, \mathbf{v}, t)$, where $i=0,1, \ldots, i_{\max }$ denotes the ion charge, and $i_{\text {max }}$ is the maximal ion charge, are:

$$
\begin{array}{r}
\partial_{t} \rho^{(e)}(\mathbf{r}, \mathbf{v}, t)+\mathbf{v} \cdot \partial_{\mathbf{r}} \rho^{(e)}(\mathbf{r}, \mathbf{v}, t)+\frac{e}{m}(\mathbf{E}(\mathbf{r}, t)+\mathbf{v} \times \mathbf{B}(\mathbf{r}, t)) \cdot \partial_{\mathbf{v}} \rho^{(e)}(\mathbf{r}, \mathbf{v}, t)= \\
=\Omega^{(e)}\left(\rho^{(e)}, \rho^{(i)}, \mathbf{r}, \mathbf{v}, t\right), \\
\partial_{t} \rho^{(i)}(\mathbf{r}, \mathbf{v}, t)+\mathbf{v} \cdot \partial_{\mathbf{r}} \rho^{(i)}(\mathbf{r}, \mathbf{v}, t)-\frac{i e}{M}(\mathbf{E}(\mathbf{r}, t)+\mathbf{v} \times \mathbf{B}(\mathbf{r}, t)) \cdot \partial_{\mathbf{v}} \rho^{(i)}(\mathbf{r}, \mathbf{v}, t)= \\
=\Omega^{(i)}\left(\rho^{(e)}, \rho^{(i)}, \mathbf{r}, \mathbf{v}, t\right) .
\end{array}
$$

These equations include the total electromagnetic force acting on ions and electrons. Collision terms, $\Omega^{(e, i)}$, describe the changes of the electron (ion) densities with time. These changes are due to short-range microscopic processes. The type of processes involved in the sample dynamics depends on the radiation wavelength.

The resulting simulation tool evolves the particle densities. Therefore the computational costs do not scale directly with the number of atoms within the sample. During the sample evolution no assumption of local thermodynamic equilibrium (LTE) is made [77]. Therefore this code can be applied to describe dynamics of samples irradiated with ultra-short pulses of the duration less than a few femtoseconds, i. e. less or comparable with the thermalization timescale.

We can use the statistical Boltzmann approach proposed in Ref. [76, 78] for describing the evolution of electron densities in solids within the valence and conduction bands. Assuming 
the homogeneous system and neglecting long term interactions, we can introduce periodic boundary conditions and reduce Eqs. (2), (3) to the ordinary integro-differential equations and follow the time evolution of the electron densities, as was done, e. g. in Refs. [79, 80]:

$$
d_{t} \rho^{(e)}(E, t)=\Omega^{(e, s o l i d)}\left(\rho^{(e)}, \rho^{(i)}, E, t\right) .
$$

The microscopic interactions such as photoabsorptions, collisional processes enter these equations as rates. These rates are included into the two-body collision terms, $\Omega^{(e, s o l i d)}\left(\rho^{(e)}, \rho^{(i)}, E, t\right)$ and are estimated either from experimental data or with theoretical models.

\section{Boltzmann approach for solids: application of simplified kinetic equation to silicon}

Here we will consider a realization of simplified Boltzmann equation known as the Multiple Rate Equation [81-85]. It turns out to be a relatively simple but quite accurate model for description of electronic behavior in semiconductors after their irradiation with a femtosecond pulse of visible light. The main idea of this approach is a discretization of the energy scale in the conduction band in steps corresponding to the mean phonon energy of the material $\Delta \varepsilon=\varepsilon_{i}-\varepsilon_{i-1}[81]$.

$$
\begin{array}{r}
\dot{n}_{i}=\dot{n}_{1 p} \theta\left(\left(\hbar \omega_{L}-E_{g}\right)-\varepsilon_{i}\right)+\dot{n}_{2 p} \theta\left(\left(2 \hbar \omega_{L}-E_{g}\right)-\varepsilon_{i}\right)+ \\
\left(W_{i-k}^{1 p h} n_{i-k} \theta\left(\varepsilon_{i}-\hbar \omega_{L}\right)-W_{i}^{1 p h} n_{i}\right)+\left(\frac{n_{i+1}}{\tau_{i+1}^{p h}} \cdot\left(1-\delta_{i, m}\right)-\frac{n_{i}}{\tau_{i}^{p h}} \cdot\left(1-\delta_{i, 1}\right)\right)+ \\
\left(\sum_{l=j}^{m} \alpha_{l} n_{l} \cdot \delta_{i, 1}+\alpha_{i+j \prime} n_{i+j \prime}-\alpha_{i} n_{i} \cdot \theta\left(\varepsilon_{i}-E_{\mathrm{e}}^{\text {min }}\right)\right)+ \\
\left(\gamma n_{k \prime}^{2} n_{\text {free }} \cdot \theta\left(\varepsilon_{i}-E_{g}\right)-2 \gamma n_{i}^{2} n_{\text {free }}\right),
\end{array}
$$

where $\dot{n}_{i}=d n_{i} / d t$ is a change of the electron density $n_{i}$ on the energy level $i$ with time $(i=1, \ldots, m)$. The first row in Eq. (5) describes the increase of electron density excited from the valence band after one-photon absorption with the rate, $\dot{n}_{1 p}$. Depending on the initial energy in the valence band, the excited electrons can reach the energy levels from the bottom of the conduction band $\varepsilon_{1}=0$ up to the maximum energy, $\hbar \omega_{L}-E_{g}$. The second term with the two-photon rate, $\dot{n}_{2 p}$, accounts in a similar way for the two-photon absorption.

The next row of Eq. (5) contains: (i) the free-electron photoabsorption terms, $W_{i}^{1 p h}$. The first photoabsorption term describes the incoming flux of electrons, while the second one is 
responsible for the loss of electrons at the respective energy level due to the photoabsorption, and (ii) the energy exchange terms (with phonons) estimated in the framework of the relaxation-time approach with the characteristic time, $\tau_{i}^{p h}$, which depends on the energy of an electron at level $i$. Since the discretization interval was chosen to be equal to the mean phonon energy $\Delta \varepsilon$, the phonon emission will lead to electron transitions between the neighboring levels $i+1$ and $i$ only.

The last two rows of Eq. (5) describe the impact ionization with the probability of $\alpha_{k}$, and the Auger recombination or three-body recombination, which are characterized by the recombination coefficient $\gamma$ and show power law dependence on the electronic density.

As discussed above, Eq.(5) does not contain spatial dependence, and, thus, neglects particle and heat transport. The model is then valid for the thin layer materials under laser irradiation, or at short subpicosecond timescales [86], when the electron transport does not play a significant role. The spatial dependence can be introduced analogously to the method presented in Ref. [84]. However, for a proper transport description, one would have to include the neglected terms from the original kinetic equation (2).

Additionally, we mention that for computational efficiency the electron-electron scattering terms were neglected here. That leads to a fast numerical realization of the algorithm. Thermalization effects can be still accounted through a relaxation-time approach, similarly to that in Ref. [87].

Equation (5) was applied to model the evolution of a solid silicon target irradiated with a femtosecond laser pulse of $625 \mathrm{~nm}$ wavelength. The detailed discussion of the results is presented in Ref. [81]. The resulting transient number of excited electrons is plotted in Fig. 10a. The reflectivity, estimated for this number of free electrons with the Drude model, shows overall good agreement with the experimental data [81], see Fig. 10b. At shorter timescales, the agreement is nearly perfect, while for the later times the agreement becomes only qualitative, which can be explained by the fact that the model does not account for the sudden changes of the reflectivity due to the melting of the target [81]. The estimated fluence for the damage threshold also coincide well with the experimental observations. Damage is understood here as any kind of phase transition (like melting or ablation), which leads to stable and observable changes in the atomic structure. For details on experimental observations of damage please see Ref. [81], where a table of the collected experimental results with the relevant references is included. 
A kinetic approach can also be used for the XUV excited solids to follow low energy electrons. In the previous section we have shown that in this case the typical electron distribution consists of two parts: a low-energy component (close to Fermi-thermalized one) and a high-energy component containing the highly excited electrons [57]. Thus, a combined model can be constructed that treats these two components with different, computationally efficient simulation methods.

\section{MODULAR, COMBINED MD/MC AND BOLTZMANN APPROACH TO STUDY STRUCTURAL CHANGES IN IRRADIATED SOLIDS}

The approaches described in the previous sections were aimed at the study of electron distributions within irradiated solids for different radiation fluence regimes and at different free electron energies. However, the picture of the irradiated solid is not yet complete: irradiation causes ionization and this triggers the rearrangement of the atomic and band structure of the solid. Previous approaches do not account for these effects, because we assumed a static, neutral atomic system with emitted electrons, which thus has an unchanging band structure.

Therefore, to resolve this deficiency we present a combined modular MD/MC and Boltzmann approach to study structural changes in irradiated solids. Dynamics of atoms and ions are then followed with an ab initio MD method. This allows one to describe changes of the bonding between atoms and ions. Simulations of structural transitions below the damage threshold, e. g. graphitization of diamond, are then accessible. MD calculations can be based on either semi-empirical potentials obtained from the fitting to experimental data or to ab-initio calculations [89], or use can be made of an ab initio DFT-MD scheme, proposed by Car and Parrinello [90], or the simplified approach of Jeschke [87].

Evolution of an electron cloud is followed with the combined MC/Boltzmann method. High energy electrons can then be described with MC code, while the dynamics of band electrons within the valence and conduction bands can be treated with a dedicated Boltzmann solver. The MC method also allows one to describe creation and relaxation of the created core holes.

At each time step, while solving the dynamics of electrons and atoms/ions, the changes of band structure and of potential energy surface are followed with a tight binding (TB) 


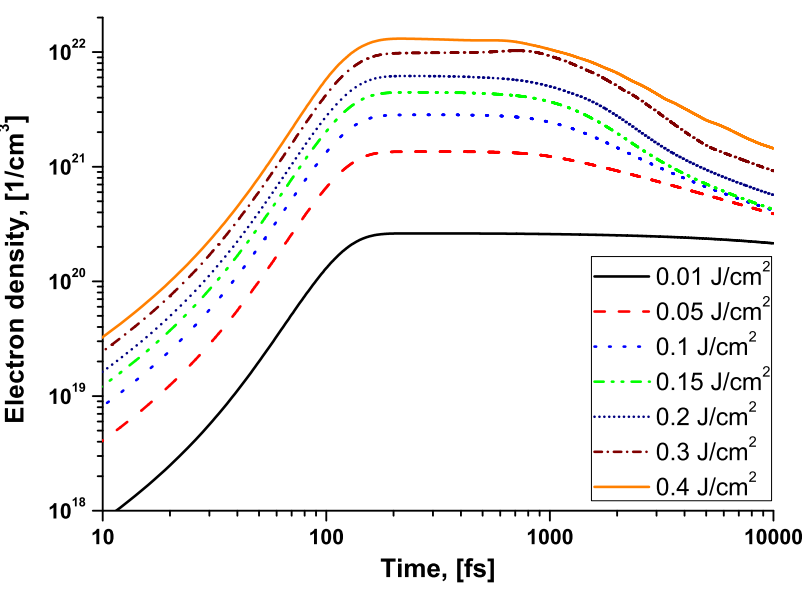

a)

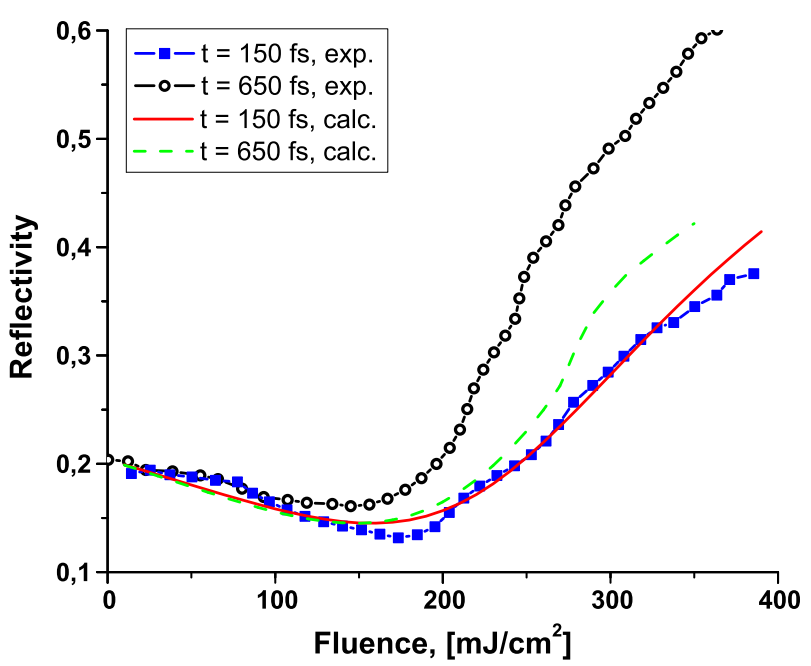

b)

FIG. 10: (Color online). (a) The total density of free electrons during and after irradiation of the solid Si target with $625 \mathrm{~nm}$ laser pulse of $150 \mathrm{fs}$ duration obtained at different pulse fluences. (b) The reflectivity as a function of fluence obtained for the same conditions for two different times; the squares and circles are experimental data from Ref. [88]; the lines are the results of the calculations. Both figures are taken from Ref. [81].

method. We use a tight-binding molecular dynamics, TBMD [87] that may also account for the changing geometry of the simulated supercell within the Parrinello-Rahman approach $[6,90]$. In the TBMD approach, the effects of the strong electronic nonequilibrium created by ultrashort laser pulses are treated by determining the time-dependent electronic distribution 
function, and, in turn, the time-dependent potential energy surfaces.

In the TBMD simulations the forces acting on atom $k$ are calculated as

$$
F_{k}(t)=-\nabla_{k} \Phi\left(r_{i j}, t\right)
$$

where $r_{i j}=r_{i}-r_{j}$ is the distance between atoms $i$ and $j$, and $\Phi\left(r_{i j}, t\right)$ is the potential energy surface, depending on all atoms in the system. The description of the laser-induced non-equilibrium processes in solids must be estimated from a microscopic electronic theory. In the framework of the TBDM, it can be written as follows:

$$
\Phi\left(r_{i j}, t\right)=-\sum_{m} n\left(E_{m}, t\right) E_{m}-\frac{1}{2} \sum_{i j} E_{r e p}\left(r_{i j}\right) .
$$

In Eq.(7) the electron energy levels, $E_{m}=\left\langle m\left|H_{T B}\left(r_{i j}\right)\right| m\right\rangle$, are the eigenvalues of the electronic TB Hamiltonian, the $n\left(E_{m}, t\right)$ is the corresponding electronic distribution function, and $E_{r e p}\left(r_{i j}\right)$ is the repulsive core-core potential.

The tight-binding Hamiltonian is written as

$$
H_{T B}=\sum_{i l} n_{i l} E_{i l}+\sum_{i l j m} t_{i j}^{l m} c_{i l}^{+} c_{j m}
$$

where $n_{i l}$ is the occupation number for the orbital $l$ of an atom $i$; and $t_{i j}^{l m}$ is the 'hopping' matrix element for the orbitals $l$ and $m$ of the atoms $i$ and $j$. The TB method assumes that the orientation dependence of the 'hopping' integrals can be separated from their distance dependence. Orientation dependence is described by Slater and Koster in Ref. [91]. The distance dependence is usually treated as a fitting parameter in TB, as well as the repulsive core-core potential, and it is chosen in order to reproduce a specific structure of a material.

However, over the last two decades the method has been extended to go beyond the static material configuration and to reproduce the dynamic changes in its geometry. This novel method is known as a transferrable tight binding, and it can be used for non-equilibrium MD simulations with a supercell of changing geometry [87].

Finally, scattering/ionization rates needed for simulations are calculated from the estimated complex dielectric function updated at each time step.

This complex code is being presently developed in our group. The current version focuses on structural transitions in FEL irradiated diamond and will be adapted to study transitions in other solids. 


\section{CONCLUSIONS}

In order to describe ultrafast transformations in solids induced by intense XFEL radiation, we need to understand the physical processes on a microscopic level. Dedicated theoretical tools are needed to follow electron and ion dynamics within the irradiated samples. The results of the simulations can then be compared to experimental data. This comparison also serves as a test of validity of different models.

Choice of the method for modelling the radiation damage depends on the structure of the sample, photon energy and pulse fluence. If one aims to follow only the electron dynamics, MC models based on the independent particle approach can deliver an accurate description, when the radiation damage, which is estimated through an average ionization degree per atom, is low. These models can be improved by including some electron correlation effects, and their validity can then be extended to the higher pulse fluences. But they are not valid at high average ionization degree, when the system transforms into a plasma. Dedicated plasma models should then be applied. However, for the applications discussed here, one aims to model the changes in solids before their structure is destroyed by radiation. So the computationally efficient MC models valid for non-highly-ionized systems are sufficiently accurate.

Following the structural changes within irradiated solids, which will include the evolution of their band structure and atomic bonding, requires following the dynamics of ions and atoms and the interaction between electronic and ionic systems. For this purpose the combined approach developed for the particle methods, i.e., MC/MD, and the kinetic method, i.e., the Boltzmann equation including the time-dependent TB description of the band structure, is a promising alternative. This tool will be able to treat a wide variety of problems and, thus, has implications for all experiments investigating transitions in solids triggered by an intense XFEL radiation. 


\section{Acknowledgments}

Authors are grateful to D. Gericke, J. Hajdu, H. Jeschke, Z. Jurek, R. W. Lee, R. London, C. Ortiz, B. Rethfeld and A. Szöke for collaboration and discussions.

[1] P. Emma, R. Akre, J. Arthur, R. Bionta, C. Bostedt, J. Bozek, A. Brachmann, P. Bucksbaum, R. Coffee, F.-J. Decker, Y. Ding, D. Dowell, S. Edstrom, A. Fisher, J. Frisch, S. Gilevich, J. Hastings, G. Hays, P. Hering, Z. Huang, R. Iverson, H. Loos, M. Messerschmidt, A. Miahnahri, S. Moeller, H.-D. Nuhn, G. Pile, D. Ratner, J. Rzepiela, D. Schultz, T. Smith, P. Stefan, H. Tompkins, J. Turner, J. Welch, W. White, J. Wu, G. Yocky, and J. Galayda. Nature Photon., 4:641, 2010.

[2] M. Altarelli, R. Brinkmann, M. Chergui, W. Decking, B. Dobson, S. Düsterer, G. Grübel, W. Graeff, H. Graafsma, J. Hajdu, J. Marangos, J. Pflüger, H. Redlin, D. Riley, I. Robinson, J. Rossbach, A. Schwarz, K. Tiedtke, T. Tschentscher, I. Vartaniants, H. Wabnitz, H. Weise, R. Wichmann, K. Witte, A. Wolf, M. Wulff, and eds. M. Yurkov. The European X-Ray Free-Electron Laser Technical Design Report, DESY, Germany, 2007.

[3] D. Pile. Nature Photon., 5:456, 2011.

[4] N. Metropolis and S. Ulam. J. of the American Statistical Association, 44:335, 1949.

[5] B. J. Alder and T. E. Wainwright. J. Chem. Phys., 31:459, 1959.

[6] A. Rahman. Phys. Rev., 136:A405, 1964.

[7] J. M. Buick. Lattice Boltzmann methods in interfacial wave modelling. Doctoral Thesis, University of Edinburgh, page 9, 1997.

[8] S. Chapman and T. G. Cowling. The mathematical theory of non-uniform gases. Cambridge Univerisy Press, 1970.

[9] V.V. Aristov. Direct methods for solving the Boltzmann equation and the study of nonequilibrium flows. Fluid Mechanics and its Applications; Kluwer Academic Publishers, 60, 2001.

[10] R. Neutze et al. Nature, 406:752-757, 2000.

[11] J. L. Quinn. Phys. Rev., 126:1453, 1962.

[12] D. R. Penn. Phys. Rev. B, 35:482, 1987.

[13] S. Samarin et al. Surf. Sci., 548:187, 2004. 
[14] L. I. Johansson and B. E. Sernelius. Phys. Rev. B, 50:16817, 1994.

[15] V. M. Silkin, E. V. Chulkov, and P. M. Echenique. Phys. Rev. B, 68:205106, 2003.

[16] I. Campillo et al.' Phys. Rev. B, 61:13486, 2000.

[17] V. P. Zhukov and E. V. Chulkov. J. Phys. Condens. Matter, 14:1937, 2002.

[18] IBM Research. Damocles home page. http://www.research.ibm.com/DAMOCLES.

[19] A. Barbieri and M.A. van Hove. Phase Shift package, http://electron.lbl.gov/leedpack/.

[20] B. Ziaja, D. van der Spoel, A. Szöke, and J. Hajdu. Phys. Rev. B, 64:214104, 2001.

[21] B. Ziaja, A. Szöke, D. van der Spoel, and J. Hajdu. Phys. Rev. B, 66:024116, 2002.

[22] B. Ziaja, R. A. London, and J. Hajdu. J. Appl. Phys., 97:064905, 2005.

[23] B.H. Bransden and C.J. Joachain. Physics of atoms and molecules. Longman, Essex, pages 505-513, 1998.

[24] P.G. Burke. Atomic, molecular and optical physics handbook. Editor G.W.F. Drake. Woodbury, New York American Institute of Physics, page 536, 1996.

[25] E. Fermi. Z. Phys., 29:315, 1924.

[26] H. Bethe. Ann. Phys. (Leipzig), 5:325, 1930.

[27] N.W. Ashcroft and N.D. Mermin. Solid state physics. Harcourt, Inc., 1976.

[28] S. Tanuma, C.J. Powell, and D.R. Penn. Surf. Interf. Anal., 17:911, 1991.

[29] D. Arnold, E. Cartier, and D.J. DiMaria. Phys. Rev. B, 49:10278, 1994.

[30] J. Lindhard. K. Dan. Vidensk. Selsk. Mat. Fys. Medd., 28:1-57, 1954.

[31] S. Tanuma, C.J. Powell, and D.R. Penn. Surf. Interf. Anal., 11:577, 1988.

[32] S. Tanuma, C. J. Powell, and D. R. Penn. Surf. Interf. Anal., 17:911, 1991.

[33] S. Tanuma, S. Ichimura, and K. Yoshihara. Appl. Surf. Scien., 100/101:47, 1996.

[34] P. Pianetta. Low-energy electron ranges in matter. X-ray data booklet, pages 3-5, 2001.

[35] T. Watanabe and et al. Jpn. J. Appl. Phys. 2, 40(7B):L715, 2001.

[36] A. Jablonski and C. Powell. NIST Standard Reference Database.

[37] M.V. Fischetti and S.E. Laux. Proceedings of the 26th European Solid State Device Research Conference (ESSDERC'96), G. Baccarani and M. Rudan Eds., Editions Frontieres Gif-surYvette Cedex, 1996, page 813, 1996.

[38] P. D. Yoder and K. Hess. Semicon. Sci. Technol., 9:852, 1994.

[39] D. K. Gautam, W. S. Khokle, and K. B. Garg. Phys. Stat. Sol.(b), 145:269, 1988.

[40] H. K. Jung, K. Taniguchi, and C. Hamaguchi. J. Appl. Phys., 79:2473, 1995. 
[41] T. Kunikiyo and et al. J. Appl. Phys., 79:7718, 1996.

[42] M. Reigrotzki and et al. J. Appl. Phys., 86:4458, 1999.

[43] M. Lorenzini and J. van Houdt. Solid-State Electron., 46:223, 2002.

[44] Database on Semiconductors. http://www.ioffe.rssi.ru/SVA/NSM/Semicond/.

[45] E. O. Kane. Phys. Rev., 159:624, 1967.

[46] Y. Kamakura et al. J. Appl. Phys. 88:5802, 2000.

[47] A. S. Barnard, S. P. Russo, and I. K. Snook. Phil. Mag. B, 82:1767, 2002.

[48] Y.Z. Chen and T.W. Tang. J. Appl. Phys., 65:4279, 1989.

[49] S. H. Gamal and T.S. Al-Harbi. Microelec. J., 32:327, 2001.

[50] S. F. Kozlov, R. Stuck, M. Hage-Ali, and P. Siffert. IEEE Trans. Nucl. Sci, NS-22:160, 1975.

[51] C. Canali et al. Nucl. Inst. Meth., 160:73, 1979.

[52] F. Nava et al. IEEE Trans. Nuc. Sci., NS-26:308, 1979.

[53] R. C. Alig, S. Bloom, and C. W. Struck. Phys. Rev. B, 22:5565, 1980.

[54] R. C. Alig. Phys. Rev. B, 27:968, 1983.

[55] K. Nishimura, J. Kawata, and K. Ohya. Nucl. Inst. Meth. Phys. Res. B, 164-165:903, 2000.

[56] J.-Ch. Kuhr and H.-J. Fitting. J. Elec. Spec. Rel. Phenom., 105:257-273, 1999.

[57] N.Medvedev, U. Zastrau, E. Förster, D.O. Gericke, and B. Rethfeld. Phys. Rev. Lett., 107:in print, 2011.

[58] N. Medvedev and B. Rethfeld. Proc. of SPIE, 8077:807716-807725, 2011.

[59] N. Medvedev and B. Rethfeld. AIP Conf. Proc., 1278:250-261, 2010.

[60] B.L. Henke, E.M. Gullikson, and J.C. Davis. At. Data and Nucl. Data Tables, 54:181-342, 1993.

[61] D.A. Papaconstantopolous. Handbook of the Band Structure of Elemental Solids. New York: Plenum, 1986.

[62] F. Ladstädter, U. Hohenester, P. Puschnig, and C. Ambrosch-Draxl. Phys. Rev. B, 70:235125$235135,2004$.

[63] M. Gryziński. Phys. Rev., 138:A305-A321, 1965.

[64] W. Lotz. Z. Physik, 216:241-247, 1968.

[65] H.-K. Chung, M.H. Chen, W.L. Morgan, Y. Ralchenko, and R.W. Lee. High Energy Density Physics, 1:3-12, 2005.

[66] A. Akkerman, J. Barak, and D. Emfietzoglou. Nucl. Instr. and Meth., 227:319-336, 2005. 
[67] M.D. Barriga-Carrasco. Phys. Rev. E, 82:046403-046408, 2010.

[68] N. Medvedev and B. Rethfeld. New J. Phys., 12:073037-073043, 2010.

[69] C.-O. Almbladh, A. L. Morales, and G. Grossmann. Phys. Rev. B, 39:3489-3502, 1989.

[70] N. Medvedev and B. Rethfeld. Europhys. Lett., 88:55001-55005, 2009.

[71] S.M. Vinko et al. Phys. Rev. Lett., 104:225001-225005, 2010.

[72] U. Zastrau, C. Fortmann, R.R. Fäustlin, L.F. Cao, T. Doppner, S. Düsterer, S.H. Glenzer, G. Gregori, T. Laarmann, H.J. Lee, A. Przystawik, P. Radcliffe, H. Reinholz, G. Röpke, R. Thiele, J. Tiggesbäumker, N.X. Truong, S. Toleikis, I. Uschmann, A. Wierling, T. Tschentscher, E. Förster, and R. Redmer. Phys. Rev. E, 78:066406-066410, 2008.

[73] D.A. Chapman and D.O. Gericke. Phys. Rev. Lett., 107:in print, 2011.

[74] Z. Jurek, G. Faigel, and M. Tegze. Eur. Phys. J. D, 29:217, 2004.

[75] A. Heidenreich, I. Last, and J. Jortner. Eur. Phys. J. D, 35:567, 2005.

[76] B. Ziaja, A. R. B. de Castro, E. Weckert, and T. Möller. Eur. Phys. J. D, 40:465, 2006.

[77] H. K. Chung et al. H. Ener. Dens. Phys., 1:3, 2005.

[78] B. Ziaja, E. Weckert, and T. Möller. Laser and Particle Beams, 25:407, 2007.

[79] B. Rethfeld et al. Phys. Rev. B, 65:214303, 2002.

[80] A. Kaiser et al. Phys. Rev. B, 61:11437, 2000.

[81] N. Medvedev and B. Rethfeld. J. Appl. Phys., 108:103112 - 103120, 2010.

[82] B. Rethfeld. Phys. Rev. Lett., 92:187401-187404, 2004.

[83] B. Rethfeld. Phys. Rev. B, 73:035101-035107, 2006.

[84] B.H. Christensen and P. Balling. Phys. Rev. B, 79:155424-155434, 2009.

[85] B. Rethfeld, O. Brenk, N. Medvedev, H. Krutsch, and D.H.H. Hoffmann. Appl. Phys. A, 101:19-25, 2010.

[86] J.K. Chen, D.Y. Tzou, and J.E. Beraun. Int. J. of Heat and Mass Transfer, 48:501-509, 2005.

[87] H.O. Jeschke, M.E. Garcia, and K.H. Bennemann. Phys. Rev. Lett., 87:015003-015007, 2001.

[88] K. Sokolowski-Tinten and D. von der Linde. Phys. Rev. B., 61:2643, 2000.

[89] M.P. Allen and D.J. Tildesley. Computer Simulation of Liquids. Oxford:Clarendon Press, 1987.

[90] R. Car and M. Parrinello. Phys. Rev. Lett., 55:2471-2475, 1985.

[91] J.C. Slater et al. Phys. Rev. B, 94:1498-1524, 1954. 